\title{
A Comparative study of Face Recognition with Principal Component Analysis and Cross-Correlation Technique
}

\author{
Srinivasulu Asadi \\ Dept of IT \\ SVEC, Tirupati \\ J.N.T.Univeristy, Anantapur \\ A.P - 517102, India
}

\author{
Dr.Ch.D.V.Subba Rao \\ Dept of CSE, \\ College of Engineering \\ S V University, Tirupati, \\ AP-517 102, India
}

\author{
V.Saikrishna \\ Dept of CSE, \\ College of Engineering \\ S V University, Tirupati, \\ AP-517 102, India
}

\begin{abstract}
Automated face recognition has become a major field of interest. Face recognition algorithms are used in a wide range of applications viz., security control, crime investigation, and entrance control in buildings, access control at automatic teller machines, passport verification, identifying the faces in a given databases. This paper discusses different face recognition techniques by considering different test samples. The experimentation involved the use of Eigen faces and PCA (Principal Component Analysis). Another method based on Cross-Correlation in spectral domain has also been implemented and tested. Recognition rate of $90 \%$ was achieved for the above mentioned face recognition techniques.
\end{abstract}

\section{General Terms}

Image Processing, Artificial Intelligence, Biometric, Signal Processing.

\section{Keywords}

Face Recognition, Principal Component Analysis, CrossCorrelation Technique.

\section{INTRODUCTION}

Face recognition is biometric identification by scanning a person's face and matching it against a library of known faces. Face recognition is defined as the identification of a person from an image of their face. Face Recognition systems can be of two types. Firstly, Face Identification: Given a face image that belongs to a person in a database and to tell whose image it is. Secondly, Face Verification: Given a face image that might not belong to the database, verify whether it is from the person it is claimed to be in the database.

The main aim of most commercial face recognition is to increase the capability of security and surveillance systems. In theory, security systems involving face recognition would be impossible to hack, as the identification process involves unique identification methods, and thus only authorized users will be accepted. Face recognition has $\mathrm{N}$ classes, where each class represents one person from $\mathrm{N}$ individuals that mean multi class classification (one person vs. all the others). Face recognition must discriminate between the subtle differences of human faces. Face recognition is performed in order to determine the identity of each face. Applications of face recognition are access control, face databases, face identification, human computer interaction, law enforcement, smart cards and multimedia management.

\section{RELATED WORK}

Currently there are many methods of biometric identification viz., fingerprint, eye iris, retina, voice, face etc. Each of these methods has certain advantages and disadvantages, which must be considered in biometrical system developing: system reliability, price, flexibility, necessity of physical contact with scanning device and many others. Selecting the certain biometrical identification method or using the multi-biometrical system can help to support these, often discrepant, requirements. Face identification can be an important alternative for selecting and developing optimal biometrical system. Its advantage is that it does not require physical contact with image capture device (camera). Face identification system does not require any advanced hardware; it can be used with existing image capture devices like web cams, security cameras etc.

Face is not so unique as fingerprints and eye iris, so its recognition reliability is slightly lower. However, it is still suitable for many applications, taking into account its convenience for user. Also it can be used together with fingerprint identification or another biometrical method for developing more security critical applications. Multi-biometrical approach is especially important for identification (1: N) systems. Identification systems are very convenient for using, because they do not require any additional security information (smart cards, passwords etc.). On the other hand, 1: N-matching routine usually accumulates False Acceptance probability, which may become unacceptable for applications with large databases.

\section{PRINCIPAL COMPONENT} ANALYSIS

PCA also known as Karhunen Loeve projection. PCA calculates the Eigen vectors of the covariance matrix, and projects the original data onto a lower dimensional feature space, which is defined by Eigen vectors with large Eigen values. PCA has been used in face representation and recognition where the Eigen vectors calculated are referred to as Eigen faces. In gel images, even more than in human faces, the dimensionality of the original data is vast compared to the size of the dataset, suggesting PCA as a useful first step in analysis. There are many approaches to face recognition ranging from the Principal Component Analysis (PCA) approach (also known as Eigen faces). Prediction through feature matching. The idea of feature selection and point matching has been used to track human motion. Eigen 
faces have been used to track human faces. They use a principal component analysis approach to store a set of known patterns in a compact subspace representation of the image space, where the subspace is spanned by the Eigen vectors of the training image set.

PCA is a useful statistical technique that has found application in fields such as face recognition and image compression, and is a common technique for finding patterns in data of high dimension. The basic goal is to implement a simple face recognition system, based on well-studied and well-understood methods. One can choose to go into depth of one and only one of those methods. The method to be implemented is the PCA (Principle Component Analysis). It is one of the more successful techniques of face recognition and easy to understand and describe using mathematics. This method involves using Eigen faces.

The first step is to produce a feature detector (dimension reduction). Principal Components Analysis (PCA) was chosen because it is the most efficient technique, of dimension reduction, in terms of data compression. This allows the high dimension data, the images, to be represented by lower dimension data and so hopefully reducing the complexity of grouping the images.

\subsection{Methodology}

\subsubsection{Data Acquisition}

A database of different image sets of faces was constructed. It's only got 2 dimensions, and the reason why one can have chosen this is so that one can provide plots of the data to show what the PCA analysis is doing at each step.

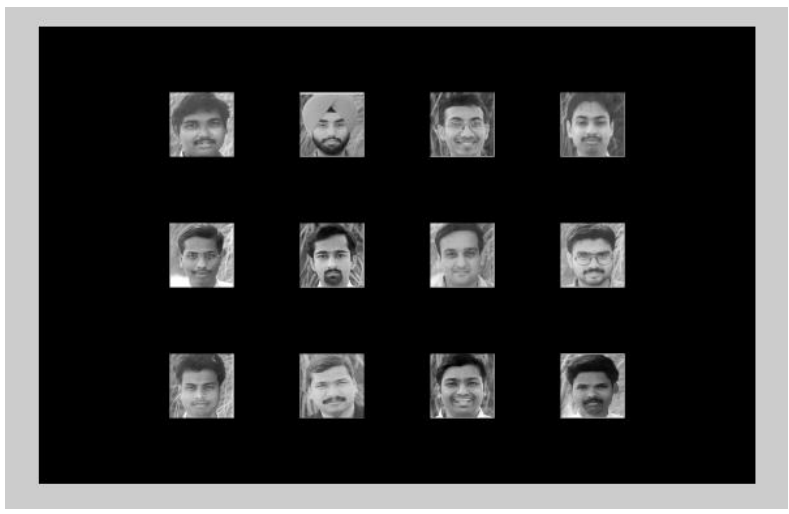

\subsection{Subtract the mean image data}

For PCA to work properly, you have to subtract the mean from each of the data dimensions. The mean subtracted is the average across each dimension. So, all the $\mathrm{x}$ values have $x^{\prime}$ (the mean of the $x$ values of all the data points) subtracted, and all the y values have y' subtracted from them. This produces a data set whose mean is zero.

\subsection{Find the covariance matrix}

This is done in exactly the same way as was discussed in section 2.2.2. Since the data is 2 dimensional, the covariance matrix will be $2 * 2$ as shown below:
Since the non-diagonal elements in this covariance matrix are positive, both the $\mathrm{x}$ and $\mathrm{y}$ variable increase together.

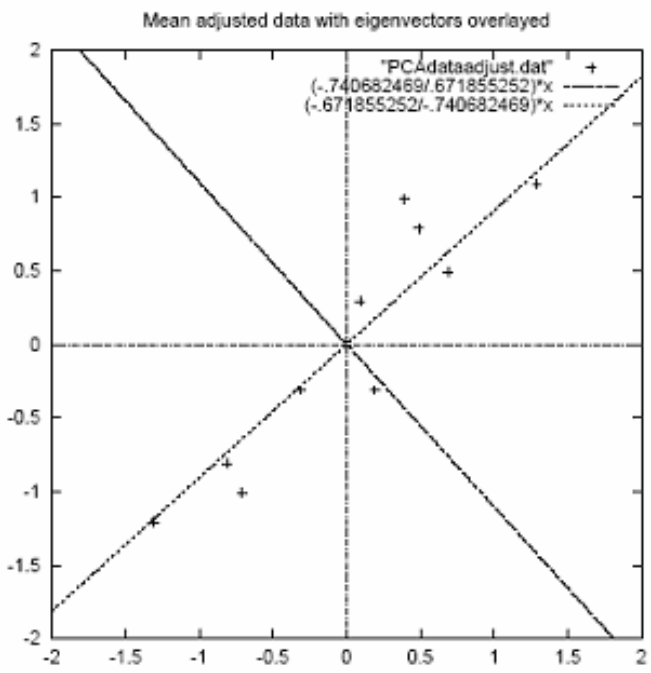

Figure 1: A plot of the normalized data (mean subtracted) with the Eigen vectors of the covariance matrix overlayed on top

\subsubsection{Recognition Process in Eigen faces Approach}

Step 1. Form a face database that consists of the face images of known individuals.

Step 2. Choose a training set that includes a number of images (M) for each person with some variation in pose and different faces.

Step 3. Calculate the $M \times M$ matrix $L$, find its Eigen vectors and Eigen values, and choose the $\mathrm{M}^{\prime}$ Eigen vectors with the highest associated Eigen values.

Step 4. Combine the normalized training set of images to produce M' Eigen faces.

Step 5. Store these Eigen faces for later use.

Step 6. For each member in the face database, compute and store a feature vector.

Step 7. Choose a threshold value e that defines the maximum allowable distance from any face class. Optionally choose a threshold $f$ that defines the maximum allowable distance from face space.

Step 8. For each new face image to be identified, calculate its feature vector and compare it with the stored feature vectors of the face library members.

Step 9. If the comparison satisfies the threshold for at least one member, then classify this face image as "known", otherwise a miss has occurred and classify it as "unknown" and add this member to the face library with its feature vector. 


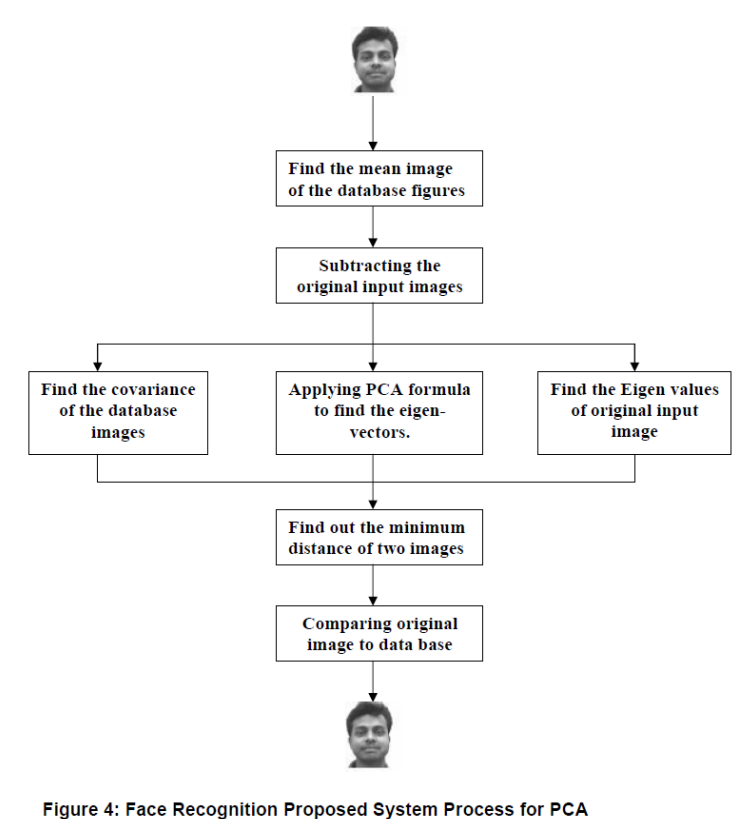

\subsection{Extracting Principal Components}

The details about the computational aspects of principal components analysis have been skipped in this section, as it has been mentioned before. (references were provided at the beginning of this section). However, basically, the extraction of principal components amounts to a variance maximizing (varimax) rotation of the original variable space. For example, in a scatter plot one can think of the regression line as the original $\mathrm{X}$ axis, rotated so that it approximates the regression line. This type of rotation is called variance maximizing because the criterion for (goal of) the rotation is to maximize the variance (variability) of the new variable (factor), while minimizing the variance around the new variable.

\subsection{Benefits of PCA}

- The basic Benefit in PCA is to reduce the dimension of the data.

- No data redundancy as components is Orthogonal.

- With help of PCA, complexity of grouping the images can be reduced.

- Application of PCA in the prominent field of criminal investigation is beneficial.

- PCA also benefits entrance control in buildings, access control for computers in general, for automatic teller machines in particular, day-to-day affairs like withdrawing money from bank account, dealing with the post office, passport verification, and identifying the faces in a given databases

\section{PCA Features}

- PCA computes means, variances, covariance's, and correlations of large data sets

- $\quad$ PCA computes and ranks principal components and their variances.

- Automatically transforms data sets.

- $\quad$ PCA can analyze datasets up to 50,000 rows and 200 columns

\section{CROSS - CORRELATION TECHNIQUE 4.1 Cross-Correlation Technique}

Correlation is often called Template matching. Correlation can be used to locate features within an image which is closely related to convolution. It is a standard of estimating the degree to which two series are correlated. The use of cross-correlation for template matching is motivated by the distance measure (squared Euclidean Distance)

Correlation of two functions $\mathrm{f}(\mathrm{x}, \mathrm{y})$ and $\mathrm{h}(\mathrm{x}, \mathrm{y})$ is defined as

$$
\begin{gathered}
\mathrm{f}(\mathrm{x}, \mathrm{y}) * \mathrm{~h}(\mathrm{x}, \mathrm{y})=1 /(\mathrm{M} * \mathrm{~N}) * \sum \mathrm{f}^{*}(\mathrm{~m}, \mathrm{n}) \mathrm{h}(\mathrm{x}+\mathrm{m}, \mathrm{y}+\mathrm{n}) \\
\mathrm{n}=0 \mathrm{~m}=0
\end{gathered}
$$

Cross-Correlation and Euclidean Distance are two of the most common statistical techniques used for target matching. Calculation of the Cross-Correlation can be carried out in both the spatial and Fourier domain. Significant performance benefits are achieved by computing the Fourier domain Cross-Correlation using the Mixed Radix Fast Fourier Transform (FFT). This paper provides further results of a comparison of these techniques undertaken in the Meteorological Product Extraction Facility environment, by comparing the displacement vectors derived from pseudo-real imagery data, including analysis of behavior in different contrast regions. The results show that the two techniques are well matched. In assessing the relative benefits of alternative matching techniques, this paper additionally provides results for the Euclidean Distance method. It includes comparison with Cross-Correlation of the displacement vectors in different contrast regions and analyses where maximum discrepancy is observed between the two methods. The results indicate that differences between the two techniques are more apparent in lower contrast regions.

\section{Matching images}

It can deal with two similar tasks:

1. Given a point in one image, to find its matching point in the other image, feature matching or optical flow.

2. Find the transformation mapping one image to the other Image alignment or Image registration.

Cross Correlation Function: The cross correlation of random processes $\mathrm{X}(\mathrm{t})$ and $\mathrm{Y}(\mathrm{t})$ is

$$
\operatorname{RXY}(t ; c)=E[X(t) Y(t+c)]
$$

A neighborhood operation in which each output pixel is a weighted sum of neighboring input pixels. The weights are defined by the computational molecule. Image processing operations are implemented with convolution which includes smoothing, sharpening, and edge enhancement. Correlation is closely related mathematically to convolution.

Issues of correlation: In many image identification processes the mask may need to be rotated and scaled at each position. The process is very similar to 2D filtering except in that case the image is replaced by 
an appropriately scaled version of the correlation. The process can be extremely time consuming. The $2 \mathrm{D}$ cross correlation needs to be computed for every point in the image.

\subsection{Implementation Method}

The Fourier transform can also be used to perform correlation, which is closely related to convolution. Correlation can be used to locate features within an image in this context [15] correlation is often called Template matching.

For instance, suppose you want to locate occurrences of the letter "a" in an image containing text. This example reads in text.tif and creates a template image by extracting a letter "a" from it,

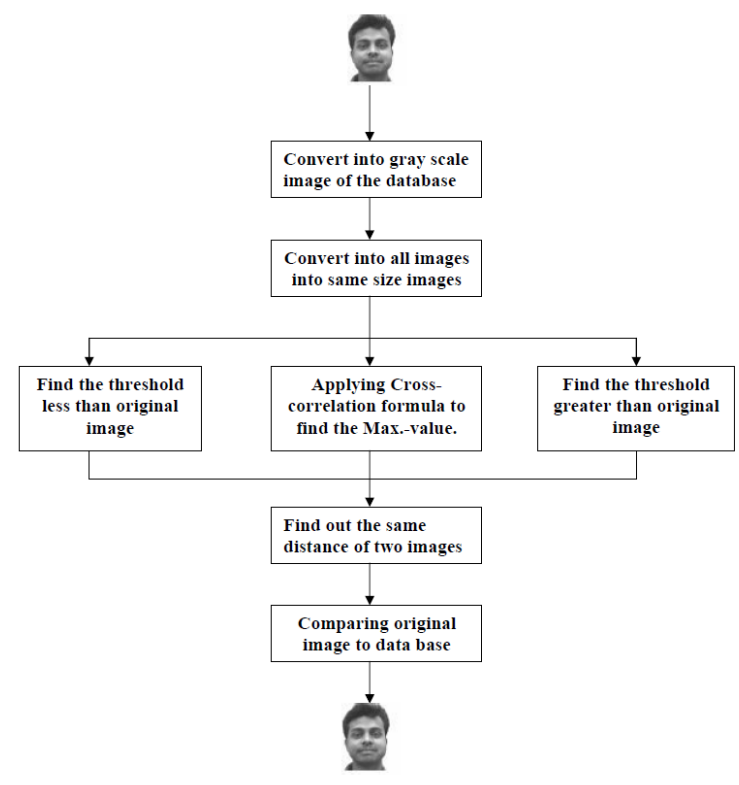

Figure 5: Face Recognition Proposed Systems for cross-correlation Technique Process

The correlation of the image of the letter "a" with the larger image can be computed by first rotating the image of "a" by $180^{\circ}$ and then using the FFT-based convolution technique[13].

To match the template to the image, you can use the $\mathrm{fft} 2$ and ifft 2 functions.

Use this formula $\mathrm{C}=\operatorname{real}(\mathrm{ifft} 2(\mathrm{fft} 2(\mathrm{bw}) *$ fft2(rot90(a,2),256,256))); calculate $\mathrm{C}$ value.

Display, scaling data to appropriate range. Figure, $\operatorname{imshow}(\mathbf{C}$, a)

Find max pixel value in $C . \max (\mathrm{C}(:))$.

Use a threshold that's a little less than max. thresh $=45$;

Display showing pixels over threshold. figure, imshow(C

$>$ thresh)

Comparing with input image to the database image set.

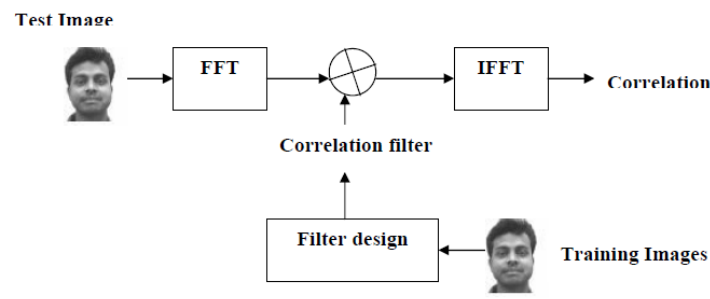

Figure 6: Block diagram showing the Correlation process

\section{RESULTS AND DISCUSSION}

The performance of the two methods was accessed by testing. The face images of 12 people with different poses (48 Images) were taken for testing. By using PCA (Principal Component Analysis) the recognition accuracy was $90 \%$ on a very small set of faces. However the accuracy might decrease with increase in the number of samples. The recognition accuracy of Eigen face method can be improved by using a Neural Network for classification rather than taking the Euclidean Distance of the features. By using Cross-Correlation Technique the recognition accuracy was $85 \%$ on a very small set of faces. However the accuracy might decrease with increase in the number of samples. The recognition accuracy of Eigen face method can be improved by using a Wavelet Networks for classification rather than taking the Euclidean Distance of the features

The results of the testing process are documented below.

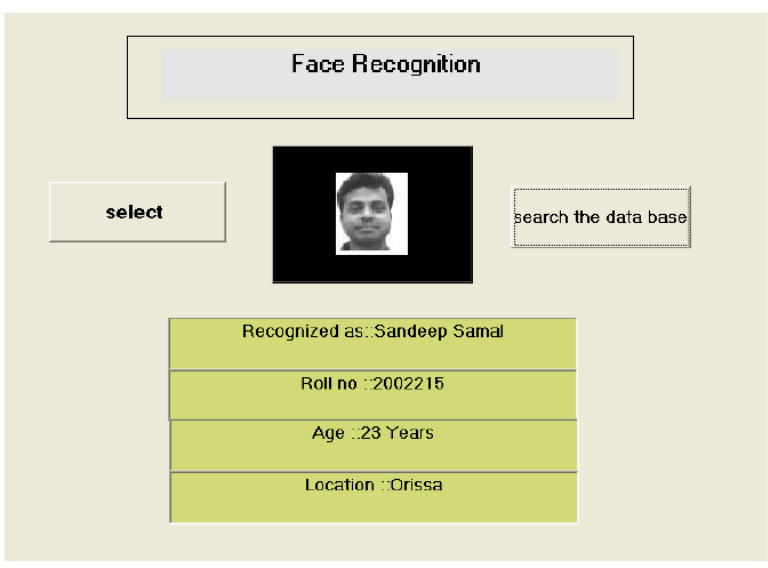

Figure 7: Recognized face using Principal Component Analysis

Principle Component Analysis (PCA), a frequently used statistical technique for optimal lossy compression of data under least square sense; provide orthogonal basis vector space to represent original data This dissertation includes research and experimentation with face recognition techniques tested with different faces principally; experimentation involved the use of Eigen faces and PCA (Principal Component Analysis). Another method based on Cross-Correlation in spectral domain has also been implemented and tested. 


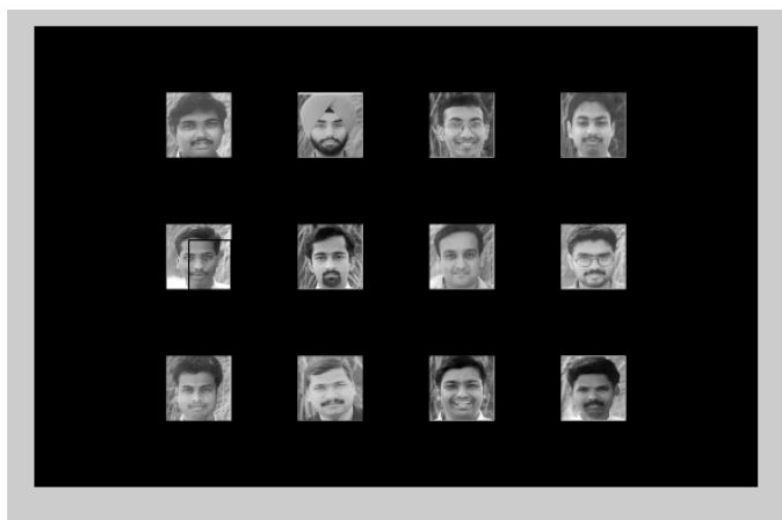

Figure 10: Recognized face using Cross-Correlation Technique

Efficiency of the Algorithm \& Suitability for Industry Use: Recognizing faces could theoretically be very fast, because it consists only of a matrix subtraction, one matrix multiplication and a bit of comparison. On the other hand, training new faces is a comparably complicated calculation, especially if the "face-space" has to be recalculated as soon as new persons are added.

\section{CONCLUSION}

Two face recognition strategies i.e. PCA (Principal Component Analysis) and Cross-correlation technique were implemented. Principal Component Analysis gave better results for varying poses. Cross-correlation technique can recognize faces even when they are rotated. But this method fails when faces with quite different poses are taken into account. The results are pretty good for the test samples that we have considered.

Face recognition is quickly emerging as a viable authentication method today. With the adoption of standards and community awareness, this technology will become more and more acceptable. Current implementations of this technology are visible in airports, ATM counters, border and security control checkpoints, crime investigation, entrance control in buildings, access control for computers in general, dayto-day affairs like withdrawing money from bank account, passport verification etc. Hence, in this contemporary world, face recognition is playing an important role and can be concluded as an upcoming technology.

Further, we can improve the system by integrating with other biometric methods like speaker recognition, finger print, eye iris, and retina and voice recognition. Further it is suggested to use neural networks and wavelet networks for improving the accuracy of Eigen face method rather than taking Euclidean distance into account

\section{REFERENCES}

[1] Jackson, J. E., "A Users Guide to Principal Components", John Wiley and Sons, pp. 1-25, 1991.

[2] Kyungnam Kim, "Face Recognition using principal component analysis", USA, June 2000.

[3] Geof Givens, J Ross Beveridge, Bruce A. Draper and David Bolme," A Statistical Assessment of Subject
Factors in the PCA Recognition of Human Faces", April 2003.

[4] Bruce A. Draper, Kyungim Baek, Marian Stewart Bartlett and J. Ross Beveridge," Recognizing Faces with PCA and ICA”, 2002.

[5] J. Ross Beveridge, Kai She and Bruce A. Draper and Geof H. Givens"A Nonparametric Statistical Comparison of Principal Component and Linear Discriminant Subspaces for Face Recognition".

[6] Matthew Turk, Alex Pentland, "Eigen faces for Recognition" Vision and Modeling Group, the Media Laboratory, Massachusetts Institute of Technology; September 1990.

[7] Imola K. Fodor," A survey of dimension reduction techniques", June 2002

[8] W. Yambor, B. Draper and R. Beveridge, Analyzing PCA-based Face Recognition Algorithms: "Eigen vector Selection and Distance Measures", July 2000

[9] Christopher James Cobb, "Face Recognition Project", December, 2001.

[10] Geof H. Givens, J. Ross Beveridge, Bruce A. Draper and David Bolme, "Using A Generalized Linear Mixed Model to Study the Configuration Space of a PCA+LDA Human Face Recognition Algorithm", April 2003

[11] B.V.K. Vijaya Kumar, "Tutorial survey of composite filter designs for optical correlators," Appl. Opt. 31, pp. 4773-4801 (1992).

[12] B.V.K. Vijaya Kumar, and D. Casasent, A. Mahalanobis, "Minimum average correlation energy filters," Appl. Opt. 26, pp. 3633-3630 (1987).

[13] Gonzales, $\mathrm{R}$ and Woods R,'Digital Image Processing, 2nd Edition Prentice-Hall Englewood cliffs, NJ, 2002.

[14] Mario's Savvides and B.V.K .Vijaya Kumar and Pradeep Khosla, "Face verification using correlation filters", U.S.A.

[15] Greg Dew and Ken Holmlund, "Investigations of Cross-Correlation of and Euclidean Distance Target Matching Techniques in the MPEF environment".

[16] Beata J Wysocki, Tadeusz A Wysocki “Orthogonal Binary Sequences with Wide Range of Correlation Properties", Australia.

[17] John W.Fisher III, "Nonlinear Extensions to the Minimum Average Correlation Energy-Filter" University of Florida, 1997.

[18] Ahmet Bahtiyar Gul "Holistic Face recognition by Dimension reduction", May 2002.

[19] Ilker Atalay, "Face Recognition using Eigen faces", January 1996.

[20] Mat lab 6.0 "Image Processing Tool Box". 